\title{
Photometric properties of young blue compact dwarf galaxy candidates
}

\author{
P. Papaderos ${ }^{1}$, Y. I. Izotov $^{2}$, N. G. Guseva ${ }^{2}$, K. G. Noeske ${ }^{3}$, \\ T. X. Thuan ${ }^{4}$ and K. J. Fricke ${ }^{1}$ \\ ${ }^{1}$ Institute for Astrophysics, Friedrich-Hund-Platz 1, 37077 Göttingen, Germany \\ ${ }^{2}$ Main Astronomical Observatory, Zabolotnoho 27, Kyiv 03680, Ukraine \\ ${ }^{3}$ Lick Observatory, University of California, Santa Cruz, CA 95064, USA \\ ${ }^{4}$ Astronomy Department, University of Virginia, Charlottesville, VA 22903, USA
}

Keywords. Galaxies: formation, evolution, dwarf, starburst, abundances, structure

A tiny fraction $(<1 \%)$ of very metal-deficient $(12+\log (\mathrm{O} / \mathrm{H}) \leqslant 7.6)$ blue compact dwarf (BCD) galaxies exhibits a nearly galaxy-wide starburst activity and no signatures of an old stellar host galaxy. The evolutionary status and formation history of these most metal-deficient BCDs are still a subject of debate. Various lines of evidence suggest, however, that these systems do not contain a substantial population of stars older than $\sim 1$ Gyr and hence qualify as nearby younggalaxy candidates. Elaborated multiwavelength studies of these rare, most metal-deficient BCDs may therefore provide crucial insights into the formation and starburst-driven evolution of lowmass galaxies in the early universe.

In particular, these extremely metal-poor BCDs constitute excellent nearby laboratories for studying high-ionization emission and the hardness of the ionizing radiation in nearly pristine extragalactic environments. If we wish to understand the spectra of primeval galaxies, it is highly important to understand how the UV radiation field of these systems changes as metallicity decreases. It is well established that the lower the metallicity, the harder is the radiation from massive stars (Campbell et al. 1986). As a consequence, strong high-ionization lines are expected in the spectra of these most chemically unevolved BCDs. Indeed, deep spectroscopy has led recently to the discovery of high-ionization emission lines, such as $[\mathrm{Fe} \mathrm{v}] \lambda 4227$ and $[\mathrm{Ne} \mathrm{v}]$ $\lambda 3346,3426$, in addition to strong He II $\lambda 4686$ emission, in a few of the most metal-deficient star-forming galaxies known in the local universe, as e.g. the young BCD candidates Tol 1214277 (Fricke et al. (2001), Izotov et al. (2004); cf. Fig. 1b, d) and SBS 0335-052 (Izotov et al. (2001); see Fig. 1c). Evidently, spectroscopic studies with large telescopes are crucial for the detection and accurate analysis of those faint spectral features.

In order to assess the photometric structure and evolutionary status of young BCD candidates it is of critical importance to correct for the effect of extended nebular emission, as the latter may severely affect colors and age estimates obtained therefrom. One such example is I $\mathrm{Zw} 18$ (Fig. 1a), the second most metal-poor emission-line galaxy known $(12+\log (\mathrm{O} / \mathrm{H})=7.17 \ldots 7.23$; Thuan \& Izotov (2005)). This system is embedded within a filamentary low-surface brightness (LSB) envelope, extending out to $18^{\prime \prime}(\sim 1.3 \mathrm{kpc}$, assuming a distance of $15 \mathrm{Mpc})$ from its starforming regions. Papaderos et al. (2002), using broad- (BVRI) and narrow-band (H $\alpha,[\mathrm{OIII}])$ HST data, have shown that the extended LSB envelope of this young BCD candidate is entirely due to nebular line emission: ionized gas accounts for more than $80 \%$ of the line-of-sight emission at a galactocentric distance of $8^{\prime \prime}(\sim 0.65 \mathrm{kpc})$ and for up to $50 \%$ of the total $R$ light of I Zw 18. Consequently, a twodimensional subtraction of ionized gas emission is indispensable for a meaningful study of the photometric structure of this system. As evident from Fig. 1b, this correction leads to the complete removal of the filamentary LSB envelope in IZw18's main body, leaving behind a very compact (exponential scale length $\alpha \approx 120 \mathrm{pc}$ ) dwarf galaxy (see Papaderos et al. (2002) for details). 

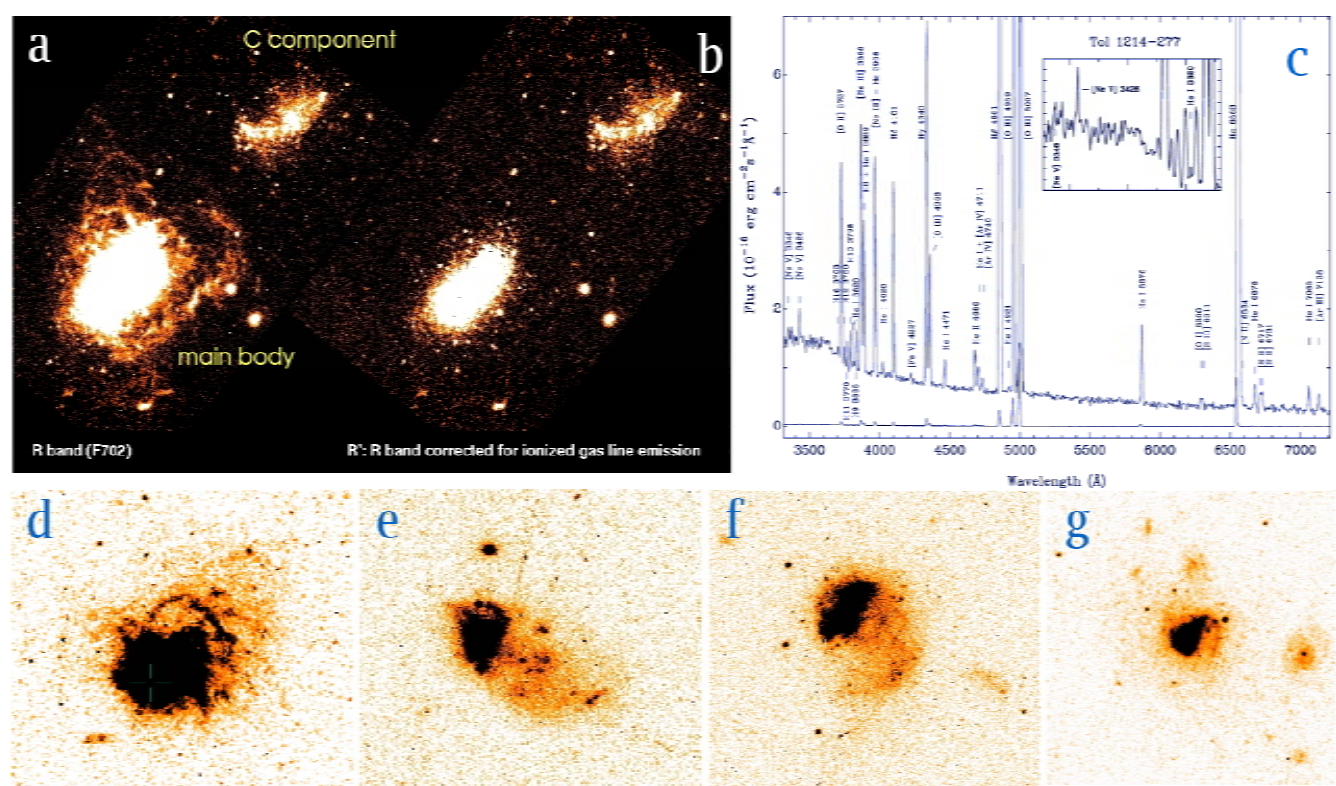

Figure 1. (a\&b) Archival HST WFPC2 exposure of I Zw 18 (D=15 Mpc) in the R(F702W) band prior to and after subtraction of nebular line emission. (c) The redshift-corrected spectrum of the brightest HII region in the low-metallicity $(12+\log (\mathrm{O} / \mathrm{H})=7.51)$ BCD Tol 1214-277 with labelled emission lines (from Izotov et al. (2004)). The lower spectrum is the observed spectrum downscaled by a factor of 50 . The inset shows a close-up view of the blue part of the spectrum with the two high-ionization [Fe V] $\lambda 4227$ and [Ne V] $\lambda 3426$ lines. (d-g) HST WFPC2 exposures of the young BCD candidates SBS 0335-052 E ( $\mathrm{D}=54.3 \mathrm{Mpc})$, Tol 1214-277 ( $\mathrm{D}=104 \mathrm{Mpc})$, Tol $65(\mathrm{D}=36 \mathrm{Mpc})$ and Pox $186(\mathrm{D}=18.5 \mathrm{Mpc})$.

Other examples of young BCD candidates with colors significantly affected by intense, spatially extended nebular line emission are SBS 0335-052E (Fig. 1d; Thuan et al. (1997), Papaderos et al. (1998), Izotov et al. (2001), Pustil'nik et al. (2004)), Tol 1214-277 (Fig. 1e; Fricke et al. (2001), Izotov et al. (2004)), Tol 65 (Fig. 1f) and Pox 186 (Fig. 1g; Guseva et al. (2004)).

\section{Acknowledgements}

N.G. Guseva and Y.I. Izotov have been supported by DFG grants 436 UKR 17/2/04 and 436 UKR $17 / 25 / 05$. P.P. greatfully acknowledges travel support from the DFG under KON 2257/2005 PA 1228/4-1 without which he could not have attended this conference.

\section{References}

Campbell, A., Terlevich, R. \& Melnick, J. 1986, MNRAS 223, 811

Fricke, K.J., Izotov, Y.I., Papaderos, P., Guseva, N.G. \& Thuan, T.X. 2001, AJ 121, 169

Guseva, N.G., Papaderos, P., Izotov, Y.I., Noeske, K.G. \& Fricke, K.J. 2004, A\& A 421, 519

Izotov, Y.I., Chaffee, F.H. \& Schaerer, D. 2001, A\&3A 378, L45

Izotov, Y.I., Noeske, K.G., Guseva, N.G., Papaderos, P. et al. 2004, A\&A 415, L27

Papaderos, P., Izotov, Y.I., Fricke, K.J., Thuan, T.X. \& Guseva, N.G. 1998, A\&6A 338, 43

Papaderos, P., Izotov, Y.I., Thuan, T.X., Noeske, K.G., Fricke, K.J., Guseva, N.G. \& Green, R.F. 2002, A\&A 292, 461

Pustilnik, S.A., Pramskij, A.G. \& Kniazev, A.Y. 2004, A\&A 425, 51

Thuan, T. X., Izotov, Y.I. \& Lipovetsky, V.A. 1997, ApJ 477, 661

Thuan, T.X. \& Izotov, Y.I. 2005, ApJS 161, 240 